\title{
GAS IN GALAXY CLUSTERS
}

\author{
Joseph Silk \\ Department of Astronomy, University of California \\ Berkeley, California 94720
}

Gaseous matter almost certainly cannot account for a significant amount of the binding mass in the cores of rich clusters. Implications of the variety of upper limits are well known (Tarter and Silk 1974), and will not be described here. However intracluster gas provides important clues to the evolution of galaxy clusters, and the present review is devoted to elucidating its role.

\section{X-RAY OBSERVATIONS}

$\mathrm{X}$-ray data have provided the principal source of evidence for the existence of a hot intracluster gas. For some time, spectral fits to the continuum in the energy range $0.2-30 \mathrm{keV}$ have marginally favored thermal as opposed to non-thermal (or power-law) intrinsic source spectra. Discovery of an emission feature at $7 \mathrm{keV}$, identified with $\mathrm{Fe} X X V$ and Fe XXVI line emission, has confirmed the presence of hot intracluster gas in four cluster sources (Mitchell et al. 1976; Serlemitsos et al. 1976; Mitchell and Culhane 1977). An unexpected finding is that the iron abundance is similar in at least three of the sources, and is generally within a factor of seven of the solar iron abundance. This latter conclusion appears to be moderately independent of various models for the gas distribution (Bahcall and Sarazin 1977). The inferred masses of gas are comparable to those within the luminous regions of the cluster galaxies, and only amount to a fraction (typically $10 \%$ in a hydrostatic model) of the cluster dynamical mass.

Recent observations with high spatial resolution (Gorenstein et al. 1977) indicate that the extended Virgo source is centered on M87 and is smal1 (core radius approximately $100 \mathrm{kpc}$ ), and may therefore be confined by the gravitational potential well of M87 itself (Mathews 1977). Several other clusters are known to be extended sources of X-ray emission, typical core-radii being several hundred kpc (Kellogg and Murray 1974), usually larger than, or comparable to, the characteristic scale of the galaxy distribution. Gas temperatures based on X-ray spectral data are now available for some fifteen cluster sources (Jones and Forman 1977); 
there is a weak correlation with X-ray luminosity, the less luminous source tending to have lower temperatures.

\section{RADIO OBSERVATIONS}

The morphology of tailed radio galaxies (TRG's) has provided dramatic evidence for the presence of intracluster gas. Most TRG's are associated with clusters; the few that are not (see below) may be associated with superclusters. There seems little doubt that the long (often curved and typically several hundred $\mathrm{kpc}$ ) tailed sources are produced and maintained by interaction with the intracluster gas (Jaffe and Perola 1973; JP). Rudnick and Owen (1974) find that two-thirds of cluster radio sources show significant amounts of distortion or misalignment, as compared with the classical double-lobe structure. Common to the various plasmoid ejection models is the confinement of the head of the TRG by ram pressure:

$$
\mathrm{p}_{\mathrm{cr}}+\mathrm{B}^{2} / 8 \pi \leqslant \frac{1}{2} \rho \mathrm{v}^{2}
$$

where $B$ is the internal magnetic field, $p_{c r}$ denotes the cosmic ray density in the tail, $\rho$ is the intracluster gas density, and $v$ is the plasmoid velocity relative to the intracluster gas. Use of the equipartition field in the expanded tail region and conservation of flux to infer $B(J P)$ yields $\rho=10^{-27} \mathrm{~g} \mathrm{~cm}^{-3}$ for 3C 129. This does not differ significantly if the ejection is subsonic (Cowie and McKee 1975), but a substantially lower value of $\rho$ was inferred in a model where reacceleration of electrons occurred in the tail (Pacholczyk and Scott 1976). In fact, this latter conclusion is erroneous, as cosmic ray pressure was neglected in applying (1). If turbulence - driven reacceleration of electrons is important and results in fields below the equipartition value in the tail, the left hand side of (1) is actually increased, and $\rho$ is larger than in the JP model. Ram pressure confinement generally leads to minimum densities $\rho \sim 10^{-27} \mathrm{~g} \mathrm{~cm}^{-3}$. This density is similar to the mean gas density found in the case of X-ray clusters. The thermal pressure of the intracluster gas is rather more model-dependent. If local acceleration occurs, no constraint can be set on the gas temperature $\mathrm{T}$. On the other hand, JP argue from the constancy of the tail width that the thermal pressure must balance the internal pressure in the tail, thereby enabling minimum pressures of $\sim 10^{-11}$ dynes $\mathrm{cm}^{-2}$ to be inferred for the intracluster gas. Tail curvature may reflect the galaxy trajectory, systematic gas flows (such as cluster winds or infall), or buoyancy in the cluster potential we11.

Are there any TRG's outside rich clusters? Jaffe (1976) cites several examples. However all are in or very near we11-known superclusters, apart from "a clear cut example of a tailed source in a sparse group of galaxies, that associated with NGC 7385". In fact, Murray et al. (1977) find a nearby X-ray source $(4 \mathrm{U} 2259+16)$ in a region of "unusually high cluster density". The known TRG's outside rich clusters might be in regions, such as superclusters, where diffuse hot gas is 
present, and thermal confinement of the tailed source is therefore possible. The equipartition pressure in the case of NGC 7385 is a factor 100 lower than found for typical TRG's in rich clusters, amounting to 10-13 dynes $\mathrm{cm}^{-2}$ (Schilizzi and Ekers 1975). This is consistent with confinement by an ambient gas of density $\sim 10^{-5} \mathrm{~cm}^{-3}$ at $\mathrm{T} \sim 10^{8} \mathrm{~K}$ (as would be implied were the X-ray source identification correct).

The correlation between decametric spectral index and presence of a radio source in an Abell cluster is well known, steeper spectral indices being found in clusters. There are indications that the $26 \mathrm{MHz}$ spectral index steepens with increasing $\mathrm{L}_{\mathrm{x}}$ (Erickson et al. 1977). The low frequency data also indicate a correlation between the $26 \mathrm{MHz}$ luminosity $\mathrm{L}_{26}$ and $\mathrm{L}_{\mathrm{X}}$ : for 14 cluster sources studied by Erickson et al., one finds $\mathrm{d} \log \mathrm{L}_{\mathrm{x}} / \mathrm{d} \log \mathrm{L}_{26}=0.5 \pm 0.3(2 \sigma)$. What perhaps is not as generally appreciated is that the higher radio frequency data show an equally good correlation. Utilizing the $1400 \mathrm{MHz}$ survey of Owen (1975), one finds that $\mathrm{d} \log \mathrm{L}_{\mathrm{x}} / \mathrm{d} \log \mathrm{L}_{1400}=0.7 \pm 0.3(2 \sigma)$ for 24 cluster sources.

\section{OPTICAL DATA}

The apparent correlation between X-ray luminosity and cluster morphological type may largely be due to a correlation between $\mathrm{L}_{\mathrm{x}}$ and cluster richness (R) (Jones and Forman 1977). The data on some $36 \mathrm{X}$-ray cluster sources can be fitted by $d \log L_{x} / d R=0.23 \pm 0.13(2 \sigma)$. Bahcall (1977a) finds a similar correlation between $\mathrm{L}_{\mathrm{X}}$ and central counts of galaxies.

Melnick and Sargent (1977) find that the radial distribution of spirals often differs from that of ellipticals and SO's in X-ray clusters. The ratio of spiral to disk systems decreases, and the elliptical fraction increases, with increasing $\mathrm{L}_{\mathrm{x}}$. The spiral fraction also decreases with increasing velocity dispersion $\Delta \mathrm{v}$. In a larger sample of 14 clusters, Bahcall (1977b) confirms that the fraction of spiral galaxies decreases with $\mathrm{L}_{\mathrm{x}}$.

The weak correlation between $\mathrm{L}_{\mathrm{x}}$ and $\Delta \mathrm{v}$ has persisted (but not strengthened) as more data has become available (Faber and Dressler 1976). One presently has, for $13 \mathrm{X}$-ray clusters with measured $\Delta \mathrm{v}$, d log $\Delta v / d$ $\log \mathrm{L}_{\mathrm{x}}=0.13 \pm 0.08(2 \sigma)$.

Another form of optical evidence for diffuse intracluster matter is due to intergalactic bridges and filaments. The diffuse light in the Coma cluster is the best studied example of this sort, but appears to largely consist of blue stars (Thuan and Kormendy 1977, and references cited therein). The interpretation of quasar absorption lines still arouses sufficient controversy that they will not be cited here as providing any unambiguous evidence for the existence of intracluster gas, theoretical arguments notwithstanding (e.g. Silk 1970). 
IV. IMPLICATIONS

The near-solar abundances of iron in at least four X-ray cluster sources appears to have eliminated pure infall models of cluster evolution; detailed spatial mapping is still needed to confirm this conclusion. One aspect of these early models has survived: infall through the gravitational potential well of the galaxy cluster remains the best candidate for supplying the thermal energy of the gas (Silk 1973). The near coincidence between cooling and Hubble times in the cluster cores can be understood if cooling regulates the amount of gas present, the gas being accreted onto the most massive, slowly moving central galaxies (and possibly initiating the formation of these systems) (Silk 1976; Cowie and Binney 1977). This could account for the frequency of occurrence of $\mathrm{cD}$ galaxies in luminous $\mathrm{X}$-ray clusters (where more gas may have initially been present). If this infall occurred sufficiently long ago, no evidence of recent star formation may remain in the galaxy colors. The dominant galaxies might actually end up being reddened if preferentially enriched material has been accreted. One might expect that the colors of $c D$ galaxies should not fall on a simple linear extrapolation of the galaxy color-magnitude relation.

The gas density in the cluster core $\propto \mathrm{T}^{1 / 2}$, whence we expect that at photon energy $\varepsilon, \mathrm{dL}_{\mathrm{x}} / \mathrm{d} \varepsilon \propto \mathrm{T}^{\mathrm{l} / 2} \exp (-\varepsilon / \tilde{\mathrm{k}} \mathrm{T})$. This relation is consistent with current data.

The correlation both of low (26 MHz) and high (1400 MHz) frequency radio fluxes with $L_{x}$ can be simply understood if the magnetic fields in the extended radio sources (that may be largely contributing to $\mathrm{L}_{\mathrm{R}}$ : Aizu 1977) originated together with the enriched intracluster gas. Assume that the relativistic electron density is maintained in equipartition with the intracluster field. This may not be implausible since local acceleration mechanisms are evidently required. Suppose that the intracluster magnetic fields and relativistic particles are initially produced by galactic supernova remnants. Since the rate at which enriched gas is produced by evolving stars should be proportional to the supernova rate, one would expect that the total cosmic ray electron energy $\mathrm{E}_{\mathrm{e}}$ is approximately proportional to the gas mass $\mathrm{M}_{\text {gas }}$. Consequently one obtains (assuming similar volumes for the $\mathrm{X}$-ray and radio sources) $\mathrm{L}_{\mathrm{R}} \stackrel{\propto}{\sim} \mathrm{L}_{\mathrm{x}}\left(\mathrm{E}_{\mathrm{el}}\right)^{2}$ $\left(\mathrm{M}_{\text {gas }}\right)^{-2}(\Delta \mathrm{v})^{-1}$. This suggests a proportionality similar to that observed (since $\left.\mathrm{L}_{\mathrm{X}} \stackrel{\propto}{\sim}(\Delta \mathrm{v})^{4}\right)$.

If equipartition is attained, the magnetic field strength should be proportional to $\mathrm{E}_{\mathrm{e}}^{1 / 2} \mathrm{r}^{-3 / 2}$ (where $\mathrm{r}$ is the source dimension). Ignoring any variation in ${ }^{2} r$, the synchrotron lifetime $t_{s}$ (at a fixed frequency) varies as $\mathrm{E}^{-3 / 4}$, or $\mathrm{M}_{\mathrm{gas}}^{-3 / 4}$ in the protogalactic infall model. Since $\mathrm{L}_{\mathrm{x}} \stackrel{\propto}{\sim} \mathrm{M}_{\mathrm{g}}^{5 / 2}$, a shorter electron lifetime $\mathrm{t}_{\mathrm{s}} \stackrel{\propto}{\sim} \mathrm{L}_{\mathrm{x}}^{-3 / 10}$ (and therefore steeper radio spectrum) is indicated in high luminosity $\mathrm{X}$-ray clusters.

The decrease of spiral content with $\mathrm{L}_{\mathrm{x}}$ and $\Delta \mathrm{v}$ supports ram pressure stripping (Gunn and Gott 1972) as the mechanism for converting spirals to So's. A quantitative estimate of its significance can be obtained in 
individual clusters where spiral galaxies that pass within a critical radius $r_{S}$ of the cluster centre (core radius $r_{c}$ ) are stripped (Tarter

\begin{tabular}{|c|c|c|c|c|c|}
\hline Cluster & $r_{s} / r_{c}$ & $\begin{array}{c}x_{\text {min }}^{2}\left(\mathrm{DF}^{*}\right) \\
\left(\mathrm{r}_{\mathrm{s}} \text { model }\right)\end{array}$ & $\begin{array}{l}x_{\mathrm{m}}^{2}\left(\mathrm{DF}^{*}\right) \\
(\mathrm{King} \\
\text { model } 1)\end{array}$ & $\left(\mathrm{km} \mathrm{s}^{-1}\right)$ & $\mathrm{r}_{\mathrm{x}} / \mathrm{r}_{\mathrm{c}}$ \\
\hline Abe11 262 & 2.4 & $0.34(7)$ & $8.8(7)$ & 760 & $(6.0)$ \\
\hline 326 & 2.0 & $0.19(9)$ & $12.5(9)$ & & \\
\hline 426 & 5.3 & $14.5(21)$ & $42.7(21)$ & 2420 & 0.91 \\
\hline 576 & 1.2 & $1.2(12)$ & $6.5(12)$ & & \\
\hline 1060 & 2.2 & $13.6(12)$ & $25.9(12)$ & 1335 & $>0.85$ \\
\hline 1656 & 5.3 & $0.16(4)$ & $6.9(4)$ & 1560 & 2.5 \\
\hline 2666 & 0.6 & $0.47(8)$ & $0.57(8)$ & & \\
\hline Virgo & 1.4 & $0.99(15)$ & $7.2(15)$ & 1070 & 0.38 \\
\hline
\end{tabular}

1977). The absence of spirals within the central regions satisfactorily fits the data of Melnick and Sargent (1977), whereas the fit to the radial distribution of spirals with a uniform (King) distribution is significantly worse (except for Abell 2666, where $r_{s}<r_{c}$ ). X-ray core radii $\left(r_{x}\right)$ generally appear to be $\hat{\imath}_{0.5} r_{s}$ (except for Abe11 262, where $r_{X}$ is poorly determined), indicating that ram pressure stripping is effective until the gas density has dropped to about $\sim 0.1$ of its central value. The ratio $r_{s} / r_{c}$ is low for three clusters with low velocity dispersion $(\Delta v)$, and larger for the two clusters with high $\Delta v$. The ram pressure stripping model predicts such correlations: $r_{s}$ should scale both with $r_{x}$, and as $\Delta v^{4 / 3}$ (for $r_{s}>r_{c}$ ). More data on $r_{x}$ and $\Delta v$ would help establish whether these indications of correlations are correct. The decrease of spiral content with $\Delta \mathrm{v}$ and $\mathrm{L}_{\mathrm{x}}$ provides further support for ram pressure stripping.

Richness is a logarithmic measure of counts of luminous galaxies within an Abell radius, and may be taken to give a measure of cluster optical luminosity $\mathrm{L}_{c \ell}$ (since cluster core radii show small variations): specifically, $R \approx 2.1$ ln $\left(L_{c \ell} / L_{O}\right)$. If we assume a relation of the form $\mathrm{d} \log \mathrm{L}_{\mathrm{x}} / \mathrm{dR}=\beta(\beta=0.23 \pm 0.13 ; \S \mathrm{III})$, then $\mathrm{L}_{\mathrm{x}} \tilde{\mathrm{e}}_{\mathrm{L}} \mathrm{L}_{\mathrm{c}} 5.1 \beta$. With $\mathrm{M}_{\mathrm{gas}} \propto$ cluster mass $\mathrm{M}_{\mathrm{c} l}$, one finds that $\mathrm{M}_{\mathrm{c} \ell} \underset{\sim}{\propto} \mathrm{L}_{x} 2 / 5 \underset{\sim}{\sim} \mathrm{L}_{\mathrm{c} l} \tilde{2}_{\beta} \underset{\sim}{\alpha} \mathrm{L}_{\mathrm{c}} \ell^{0.46}$. Other functional fits to $\mathrm{L}_{\mathrm{x}}(\mathrm{R})$ also yield a weak dep̃endence of $\mathrm{M}_{\mathrm{c} l}$ on $\mathrm{L}_{\mathrm{c} l}$. Hence $\mathrm{M}_{\mathrm{cl}} / \mathrm{L}_{\mathrm{cl}}$ decreases with increasing richness, and $\Delta \mathrm{v} \underset{\sim}{\propto}(1.6) \beta R$. Measurement of cluster velocity dispersions (and masses) would enable us to verify whether $M_{\text {gas }} \propto M_{c l}$ for $X$-ray clusters where $M_{\text {gas }}$ cannot be determined directly.

The available data (for five sources) is consistent with $\mu(\Delta v)^{2 / 3} \approx$ $\mathrm{kT}$ (where $\mu$ is the mean molecular weight), expected in most thermal models (Silk 1973). The weak $\left(\mathrm{L}_{\mathrm{X}}, \Delta \mathrm{v}\right)$ correlation (Solinger and Tucker 
1972) is presently incapable of distinguishing between different theoretical models; this situation may change when improved X-ray source size data becomes available, and can be incorporated into a search for a more general correlation.

Inverse Compton X-radiation, produced by scattering of the microwave background radiation by relativistic electrons, seems to account only for a small fraction of the observed fluxes, since the iron-line data and limits on hard $\mathrm{X}$-ray fluxes indicate that most of the emission must be thermal. One would also expect that the correlation between $\mathrm{L}_{\mathrm{x}}$ and $\mathrm{L}_{\mathrm{R}}$ should improve towards lower radio frequencies (Harriss and Romanishin 1974). A correlation between $L_{x}$ and $n$ (Gould and Raphaeli 1977), utilizing the predicted decrease in the microwave background radiation towards rich clusters (Sunyaev and Zel'dovich 1972), should be potentially capable of directly discriminating between and non-thermal models; however it is presently inconclusive, recent results (Lake and Partridge 1977; Rudnick 1977) having failed to confirm earlier indications of a positive effect (Gull and Northover 1976) in known X-ray clusters. There are indications, however, of the "cooling" effect in very rich clusters.

Nevertheless, inverse Compton X-rays are bound to be important in nearby clusters at photon energies $\approx 30 \mathrm{keV}$, and also in distant clusters at $z \gtrsim 1$ (e.g. Rees 1967). Assuming equipartition field strengths, the ratio of inverse Compton to thermal $\mathrm{X}$-ray fluxes is $\mathrm{L}_{\mathrm{X}}^{\mathrm{ic}} / \mathrm{L}_{\mathrm{X}}^{\text {th }} \approx 0.01(1+z)^{4}$ $\left[M_{\text {gas }}(z) / M_{\text {gas }}(0)\right]^{-1}$. If the gas mass does not change with time, nonthermal $\mathrm{X}$-rays will dominate at $\mathrm{z} \approx 1.5$. The gas mass cannot exceed the dynamical cluster mass, typically $\sim 10 \mathrm{M}_{\text {gas }}(0)$ : hence inverse Compton $\mathrm{X}-$ rays must dominate at $\mathrm{z} \sim 4$.

\section{ORIGIN AND EVOLUTION}

The gradual increase of the elliptical to disk galaxy content of clusters with increasing $\mathrm{L}_{\mathrm{x}}$ and decreasing Bautz-Morgan type suggests that the morphological distribution of galaxy types may be determined when the cluster forms. This is not a unique interpretation, but a possible explanation may be that galaxies undergo strong mutual interactions when clusters form. This can only occur to any significant extent if the galaxies are themselves newly formed protogalaxies. Thus we come to one of the crucial unresolved issues of galaxy formation theories: do galaxies form long before, or more or less coevally with, clusters?

The gas content of clusters leads support to the notion of coeval formation. The amount of enriched intracluster gas attests to its protogalactic origin: current mass loss rates fail to account for it. If galaxies formed in isolation, one might expect the stellar content to rapidly dominate over the gas content as star formation proceeds, just as happens when the disks of spiral and so galaxies are formed. In rich clusters, this process cannot have occurred, for some $10^{11} \mathrm{M}_{\odot}$ per galaxy is present in the form of enriched intracluster gas. This amount of matter is comparable to a typical disk mass. While ordinary spirals 
develop in isolation, it seems plausible that cluster galaxies must be inhibited from making enriched stellar disks by interacting with neighboring galaxies (cf. Ostriker 1977). One can account for intracluster gas if the enriched matter produced by ordinary stellar evolution in protogalaxies stays in gaseous form (De Young 1977): only if coeval formation of galaxies and clusters occurs does this seem easy to arrange.

There are several mechanisms by which protogalactic interactions can affect galactic evolution. The most important may be due to collisions, which will be frequent between protogalaxies in newly formed clusters (Silk 1977). Initially, relative encounter velocities may be low, and coalescence can occur. Once protogalaxies have fallen through the cluster potential well, after roughly a crossing time $\left(\sim 10^{9} \mathrm{yr}\right)$ has elapsed, the encounter velocities in rich clusters will be large compared to the internal motions within the protogalaxies. While the protogalaxies are largely gaseous, subsequent collisions will be highly disruptive. Diffuse protogalactic gas will be shock heated, and escape from the protogalaxies, but dense clouds will cool effectively, and remain bound to the protogalaxy. If the clouds are initially near the Jeans mass, the shock passage will trigger star formation.

Star formation will result in the production of enriched material, which will be ejected by supernova-driven wind flows from the less massive galaxies into the intracluster medium. Evaporation (Cowie and Songaila 1977) and ram pressure may also play a role. More massive galaxies will be capable of retaining supernova ejecta, and make successive generations of stars. Galaxies of mass $\lesssim 10^{11} \mathrm{M}_{\odot}$ may provide most of the intracluster gas, and should be relatively unenriched (and bluer) compared to much more massive systems. The metal abundance of gas in the cluster core is expected to approach that of the most massive cluster galaxies.

How can these speculations be verified? X-ray cluster galaxies should have undergone more recent star formation than other clusters, and this should be reflected in their colors, particularly at $z \geq 1$, where the cluster gas fraction (and therefore infall and ram pressure effects) may be enhanced. The color changes should exceed those expected on the basis of theories of the evolution of isolated galaxies. $L_{x}$ should be larger by a factor $\sim$ (present gas fraction) ${ }^{-2}$. Measurements of the spatial distribution of line emission will help elucidate the role of galaxy interactions. To distinguish between theoretical models, a multiparameter analysis is needed that incorporates the principal $\mathrm{X}$-ray, optical, and radio parameters. This should become feasible with the new generation of X-ray satellites.

I am indebted to Drs. J. Binney, J. Hutchins, C. F. McKee, and J. Tarter for valuable discussions, to many colleagues for providing preprints of recent results, and to NASA and NSF for research support. 


\section{REFERENCES}

Aizu, K. 1977, Pub. Astron. Soc. Japan, 29, 33.

Bahcal1, J. N. and Sarazin, C. L. 1977, Astrophys. J. Letters, 213, L99. Bahca11, N. A. 1977a, Astrophys. J. Letters (submitted).

Bahcal1, N. A. 1977b, Astrophys. J. Letters (submitted).

Cowie, L. L. and Binney, J. 1977, Astrophys. J., 215, 723.

Cowie, L. L. and McKee, C. F. 1975, Astron. and Astrophys., 63, 337.

Cowie, L. L. and Songaila, A. 1977, Nature, 266, 501.

DeYoung, D. S. 1977 (preprint).

Erickson, W. C., Mathews, T. A., and Viner, M. R. 1977 (preprint).

Faber, S. M. and Dressler, A. 1976, Astrophys J. Letters, 210, L65.

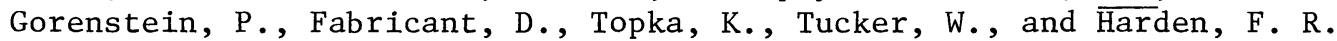
1977, Astrophys. J. Letters (submitted).

Gould, R. G. and Rephaeli, Y. 1977 (preprint).

Gul1, S. R. and Northover, K. J. E. 1976, Nature, 263, 572.

Gunn, J. E. and Gott, J. R. 1972, Astrophys. J., $1 \overline{76}, 1$.

Harriss, D. E. and Romanishin, W. 1974, Astrophys. J., 188, 209.

Jaffe, W. 1976, I. A. U. Symposium No. 74 (in press).

Jaffe, W. and Perola, G. C., Astron. and Astrophys., 26, 423.

Jones, C. and Forman, W. 1977 (preprint).

Kellogg, E. and Murray, S. 1976, Astrophys. J. Letters, 193, L37.

Lake, G. and Partridge, R. B. 1977 (preprint).

Mathews, W. G. 1977 (preprint).

Melnick, J. and Sargent, W. L. W. 1977, Astrophys. J., 215, 601.

Mitchel1, R. J. and Culhane, J. L. 1977, Monthly Notices Roy. Astron. Soc., 178, 75P.

Mitchel1, R. J., Culhane, J. L., Davison, P. J. N., and Ives, J. C.

1976, Monthly Notices Roy. Astron. Soc., 175, $29 \mathrm{P}$.

Murray, S., Forman, W., Jones, C., and Giac $\overline{\operatorname{con} i,}$ R. 1977, Astrophys. J. Letters (submitted).

Ostriker, J. P. 1977, in The Evolution of Galaxies and Stellar Popula-

tions, ed. B. M. Tinsley and R. B. Larson (Yale Univ. Press), 369.

Owen, F. N. 1975, Astrophys. J., 195, 593.

Pacholczyk, A. G. and Scott, J. S. 1976, Astrophys. J., 203, 313.

Rees, M. J. 1967, Monthly Notices Roy. Astron. Soc., 137, 429.

Rudnick, L. 1977 (private communication).

Rudnick, L. and Owen, F. N. 1977, Astron. J., 82, 1.

Schilizzi, R. T. and Ekers, R. D. 1975, Astron. and Astrophys., 60, 221.

Serlemitsos, P. J., Smith, B. W., Boldt, E. A., Holt, S. S., and $\overline{\text { S̆}} w a n k$,

J. H. 1977, Astrophys. J. Letters, 211, L63.

Silk, J. 1972 , Astrophys. J., 172, 563.

Silk, J. 1973, Ann. Revs. Astron. and Astrophys., 11, 269.

Silk, J. 1976, Astrophys. J., 208, 646.

Silk, J. 1977, Astrophys. J. (submitted).

Solinger, A. B. and Tucker, W. H. 1972, Astrophys. J. Letters 175, L107.

Sunyaev, R. A. and Zel'dovich, Ya. B. 1970, Astrophys. Sp. Sci., ㄱ, 3.

Tarter, J. 1977 (private commumication).

Tarter, J. and Silk, J. 1974, Quart. Jour. Roy. Astron. Soc., 15, 122.

Thuan, T. X. and Kormendy, J. 1977 (preprint). 


\section{DISCUSSION}

Gisler: Further evidence with regard to the ram pressure stripping of cluster galaxies may be obtained by looking at the gas content of cluster galaxies. I have just completed a study of emission line frequencies in a large sample of optical galaxy spectra, obtained from a literature survey covering the last twenty years (approximately 1300 galaxies), and I have found that the frequency of the emission line objects is indeed much lower in the Zwicky compact clusters than in less compact associations (Gisler 1977, submitted to Mon. Not. R. astr. Soc.).

Abezz: What is the basis of the $10^{14} \mathrm{M}_{\odot}$ of evolved matter that had to be ejected?

Silk: X-ray observations, in particular of the Perseus and Coma clusters, can be fitted satisfactorily by a range of thermal models that determine the total mass of X-ray emitting gas to within a factor or roughly 3 . In the simplest isothermal models, the central density is typically $\sim 3 \times 10^{-3} \mathrm{~cm}^{-3}$, and the $\mathrm{X}$-ray core radius is $\sim 0.5 \mathrm{Mpc}$.

Ostriker: Incidentally, in the galactic evolution models, you get about $10 \mathrm{M}_{\odot}$ ejected per unit solar luminosity and hence if the luminosity of the cluster is $10^{13} \mathrm{~L}_{\odot}$, you get $10^{14} \mathrm{M}_{\odot}$ ejected.

Tinsley: There is plenty of evidence for blue galaxies in clusters in the redshift interval 0.5 to 1.0 which will be discussed in my talk on Thursday.

Parijskij: I would like to mention our new results which we have obtained concerning the Sunyaev-Zeldovich effect in the Coma cluster. We first observed a small dip in the microwave background radiation in the direction of the Coma cluster in 1970 and several later observations give similar positive results but with large errors. Our latest observations were made with the 600-metre RATAN radio telescope at a wavelength of $4 \mathrm{~cm}$. We have again observed a dip in the direction of the Coma cluster having $\Delta \mathrm{T} / \mathrm{T}=-2 \times 10^{-4} \pm 0.7 \times 10^{-4} \mathrm{~K}$ and we have an estimate of its angular size. It is somewhat less than the size of the $X$-ray source in the cluster but we are not certain if the difference is significant or, if real, whether it is due to a temperature or a density effect.

Boynton: This result for the Coma cluster is consistent with zero at the two-sigma level and is therefore consistent with the very recent measurement of $\mathrm{G}$. Lake and R. B. Partridge at $\lambda=9 \mathrm{~mm}$ :

$$
\Delta \mathrm{T} / \mathrm{T}<0.05 \pm 0.05 \text {. }
$$

However, this latter limit implies no significant indication of the Sunyaev-Zeldovich effect at an even lower level of significance for this particular cluster. 
Zeldovich: Observers should also try to see the effect beyond the maximum of the spectrum of the microwave background radiation where a corresponding small increase in the temperature of the radiation in the direction of the Coma cluster should be observed. It is, however, a very difficult observation but we need to be sure about it! 